\title{
Break-off capillary tube method for blood counts
}

\begin{abstract}
S. M. LEWIS AND H. BENJAMIN From the Department of Haematology, Postgraduate Medical School, London, and Harshaw Chemicals, Daventry
\end{abstract}

The use of capillary blood for blood counts has advantages, particularly in children, and should give results little or no different from those obtained with venous blood, provided there is a free flow of blood from an adequate skin puncture. Drawing up the correct amount of blood (usually $0.02 \mathrm{ml}$.) in a micropipette requires technical skill which may be put under strain, especially with a restless patient. A simple substitute which is easy to manipulate and obviates the need for cleaning of pipettes is the use of a capillary tube cut to size so as to contain the exact volume of blood when completely filled. The capillary is then dropped into a test tube containing an appropriate volume of diluent solution. This convenient method of blood collection and dilution is particularly suitable as a bedside or field technique, but hitherto it has been of limited value owing to the difficulty in preparing capillaries of exact volume.

A further disadvantage was the presence of contaminant blood dried onto the outside of the capillary where it had been in contact with the flow, while attempts to wipe off this excess might result in the loss of a portion of the blood contained within the capillary.

\section{BREAK-OFF CAPILLARY TUBE}

By a continuous-drawing process capillary tubing can now be manufactured economically with uniform bore and free from measurable taper. Accordingly, as the volume is constantly proportional to the length, tubes of required capacity can be obtained, and it is possible to ensure an accuracy within narrow limits of tolerance (Table I). By providing a length of tubing greater than that necessary for the required volume and an accurately determined break-off point it is possible to avoid contamination of the exterior of the portion of the tube which will be placed in the diluent (Fig. 1). Length $A B$ provides a tube with a capacity of $0.02 \mathrm{ml}$., and $\mathrm{BC}$ is a length greater than that of $\mathrm{AB}$. To facilitate subsequent break-off $B$ is scored and the site of the score is indicated by a mark painted on the non-calibrated portion of the tube just beyond that point.

Received for publication 11 February 1965.

\section{1}

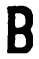

FIG. 1
TABLE I

VARIATION IN CAPACITY OF CAPILLARIES AS DETERMINED BY WEIGHT OF CONTAINED $\mathrm{H}_{2} \mathrm{O}^{1}$ Capacity of Capillary

\begin{tabular}{|c|c|}
\hline Mean & $\begin{array}{l}0.0198 \\
0.0200 \\
0 \cdot 0199 \\
0.0197 \\
0.0197 \\
0.0195 \\
0.0198 \\
0.0199 \\
0 \cdot 0198 \\
0 \cdot 0196 \\
0 \cdot 0200 \\
0 \cdot 0198 \\
0.0198\end{array}$ \\
\hline $\begin{array}{l}\text { Maximum deviations } \\
\text { From mean: } \\
\text { From reputed volume: }\end{array}$ & $\begin{array}{l} \\
+0.0002(1.0 \%) ;-0.0003(1.5 \%) \\
+0 ;\end{array}$ \\
\hline
\end{tabular}

${ }^{1}$ The reputed volume was $0.02 \mathrm{ml}$.

\section{COLLECTION OF BLOOD SAMPLE}

End $\mathrm{C}$ is brought into contact with the source of blood. When sufficient blood has been drawn up, by capillary attraction, to reach $B$ the tube is inverted so that the blood flows down and completely fills $\mathbf{A B}$. The tube is snapped at $\mathrm{B}$ and the portion $\mathrm{AB}$ is dropped into a test tube containing a measured volume of the appropriate diluent solution. This is shaken vigorously to ensure that all the blood escapes into the diluent.

This procedure provides a method for blood dilution with an accuracy in routine practice at least equal to that obtained by the use of BSS certified micropipettes (Table II). It can, of course, also be used as a speedy alternative to the pipetting of blood from a sequestrene bottle.

\section{TABLE II}

COMPARISON OF OPTICAL DENSITY READINGS OF 0.02-ML. VOLUMES OF A BLOOD SAMPLE

(DILUTED 1 IN 250)

\begin{tabular}{ll} 
Micropipettes (BSS) & Break-off Capillaries \\
\hline $36 \cdot 5$ & $36 \cdot 8$ \\
$37 \cdot 5$ & $37 \cdot 0$ \\
$36 \cdot 8$ & $36 \cdot 8$ \\
$37 \cdot 0$ & $36 \cdot 8$ \\
$37 \cdot 0$ & $37 \cdot 5$ \\
$38 \cdot 0$ & $36 \cdot 8$ \\
$38 \cdot 5$ & $37 \cdot 2$ \\
$36 \cdot 8$ & $36 \cdot 8$ \\
$37 \cdot 0$ & $36 \cdot 8$ \\
$38 \cdot 0$ & $37 \cdot 5$ \\
$38 \cdot 0$ & $36 \cdot 8$ \\
$36 \cdot 8$ & $37 \cdot 2$ \\
$37 \cdot 3$ (S.D. \pm 0.63$)$ & $37 \cdot 0($ S.D. $\pm 0 \cdot 28)$ \\
Mean & \\
Maximum deviations: & $0 \cdot 5(1 \cdot 3 \%) ;-0.2(0 \cdot 5 \%)$ \\
$+1.2(3 \cdot 2 \%) ;-0.8(2 \cdot 1 \%)$ & $+0.5(1.3 \%)$
\end{tabular}

By providing more than one break-off point in a single length of capillary tubing it is possible to speed up the process of adding equal volumes of the same blood sample to more than one diluent tube. Further work is in progress to assess the value of incorporating an anticoagulant into the capillary in order to develop a procedure for satisfactory collection of blood for platelet counts. 